\title{
Epidemiology and molecular typing of an outbreak of tuberculosis in a hostel for homeless
}

\author{
men
}

\author{
A M Kearns, A Barrett, C Marshall, R Freeman, J G Magee, S J Bourke, M Steward
}

\begin{abstract}
Aim-To investigate a possible outbreak of tuberculosis in a hostel for homeless men using IS6110 profiling, a polymerase chain reaction (PCR) based fingerprinting technique.

Methods-Eight cases of tuberculosis were diagnosed in residents of the hostel over a period of 28 months. To provide epidemiological data, a heminested inverse PCR (HIP) assay targeting the insertion sequence IS6110 together with its upstream flanking region was used to fingerprint the eight isolates of $M$ tuberculosis under investigation.

Results-The HIP technique gave IS6110 profiles which showed that while three isolates were clearly distinct, the remaining five strains were indistinguishable, suggesting the latter were representatives of a single outbreak strain.

Conclusions-The HIP assay proved discriminatory and facilitated repeated testing for the direct comparison of strains as more patients presented over the protracted course of this outbreak. (F Clin Pathol 2000;53:122-124)
\end{abstract}

Molecular Biology Unit and Regional Centre for

Mycobacteriology,

Public Health

Laboratory, General

Hospital, Westgate

Road, Newcastle upon

Tyne NE4 6BE, UK

A $M$ Kearns

A Barrett

C Marshall

R Freeman

J G Magee

Department of Respiratory Medicine, Royal Victoria

Infirmary, Newcastle upon Tyne NE1 4LP, UK

S J Bourke

Novocastra

Laboratories Ltd,

Benton Lane,

Newcastle upon Tyne

NE12 8EW, UK

M Steward

Correspondence to:

Professor Freeman

Accepted for publication

13 September 1999 typing; IS6110

An increase in the homeless population has played a significant role in the recent rise in notifications of tuberculosis. ${ }^{1}$ Contact tracing, treatment, and follow up present unique problems in this group of patients. Indeed there are no reliable figures for the prevalence or incidence of tuberculosis among the homeless in the United Kingdom. ${ }^{2}$

The need for accurate and reliable typing of Mycobacterium tuberculosis isolates is self evident, particularly in high risk groups where newly acquired infections may occur against a background of reactivation tuberculosis. Molecular typing using repetitive DNA sequences has been applied extensively in epidemiological investigations, with considerable success. ${ }^{3}$ One such method is the heminested inverse polymerase chain reaction (HIP) assay described by Patel et al, ${ }^{4}$ which targets part of the insertion sequence IS6110 and the upstream flanking region, found in organisms of the $M$ tuberculosis complex. In this study, the HIP assay was used to fingerprint eight isolates of $M$ tuberculosis involved in a cluster of cases of tuberculosis associated with a hostel for homeless men.
Keywords: tuberculosis; epidemiology; molecular

\section{Methods}

EPIDEMIOLOGY

In line with local policy, the isolation of $M t u$ berculosis from a single, smear negative sputum sample provided by an elderly vagrant suffering "respiratory difficulty" prompted an epidemiological investigation. Contact tracing was implemented but was complicated by the patient's homeless state. Investigations centred on a hostel for homeless men which he had frequented. This population group presents unique problems in that they are suspicious of authority, uncooperative, and often move between hostels and other residences. All potential contacts successfully traced were referred for clinical and bacteriological examination.

\section{BACTERIOLOGICAL EXAMINATION}

Samples were cultured using solid (Löwenstein-Jensen) and liquid (MB/BacT: Organon Teknika) mycobacterial growth media, incubated at $36^{\circ} \mathrm{C}$ for a minimum of eight weeks. Isolates of mycobacteria were confirmed by acid fast microscopy and identified using standard phenotypic methods ${ }^{5}$ and nucleic acid probes (Accuprobe; Gen-Probe Inc).

MOLECULAR TYPING

Molecular typing of phenotypically indistinguishable $M$ tuberculosis isolates was performed after the method described by Patel et al, ${ }^{4}$ with DNA extracted using the QIAamp protocol for the isolation of genomic DNA from Mycobacterium species (Qiagen). The amplification was performed on a Rapidcycler (BioGene) and the nested PCR products were visualised by ethidium bromide staining after electrophoresis through a $2 \%$ agarose gel. The resulting banding profiles were compared by eye. Reproducibility of the method was established by repeated testing of the stored DNA extracts.

\section{Results}

This hostel provides accommodation in single and double rooms for homeless men on an occasional or semipermanent basis. In common with other establishments of this type, communal facilities are provided for cooking, laundry, and recreation. Thirty four men were resident in the hostel at this time, and a further 44 previous residents were thought to be at risk. The investigation continued for 28 months, and 38 of the 78 potential contacts were traced and accepted a referral for screening. Respiratory microbiology was undertaken in those individuals showing significant radiological changes. 
Table 1 Details of eight cases of tuberculosis associated with a hostel for homeless men

\begin{tabular}{lllllllll}
\hline Case & 1 & 2 & 3 & 4 & 5 & 6 & 7 & 8 \\
\hline Age (years) & 77 & 49 & 56 & 47 & 58 & 39 & 57 & 56 \\
Date of sample & Aug 95 & Oct 95 & Nov 95 & Aug 96 & Dec 96 & Jul 97 & Dec 97 & Dec 97 \\
Smear & Negative & Negative & Negative & Positive & Positive & Positive & Positive & Positive \\
Specimen & Sputum & BAL & Sputum & Sputum & Sputum & Sputum & BAL & Sputum \\
\hline
\end{tabular}

BAL, broncho-alveolar lavage.

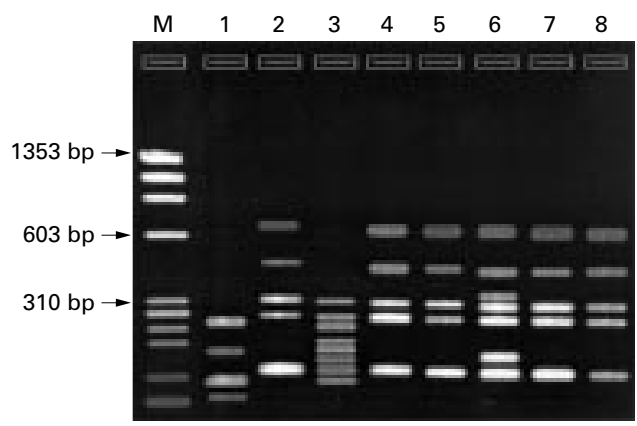

Figure 1 Heminested inverse PCR analysis of eight isolates of $M$ tuberculosis (lanes 1-8). Lane M, molecular size marker ( $\phi X 174 \mathrm{RF} D N A /$ HaeIII fragments).

Eight cases of active tuberculosis were identified, including the initial patient. All had a history of alcohol misuse and two showed evidence of old calcified lesions on chest $x$ ray, but none had previously received antituberculous treatment. All patients were started on a standard antituberculous regimen. Four died during the treatment period (two deaths being attributed directly to tuberculosis). The remaining four (including the initial case) successfully completed treatment and were discharged from further care.

Microbiological details of all eight cases are shown in table 1 . Standard phenotypic profiling including drug susceptibility testing showed all mycobacterial isolates to be $M t u$ berculosis and to be indistinguishable by these indices.

Initial RFLP typing, using standard methods, ${ }^{6}$ of the first three isolates obtained (data not shown) generated profiles with too few bands for valid interstrain comparison. Subsequently, these three isolates and a further five were examined in our laboratory by HIP profiling, and the results are shown in fig 1 . The first isolate was clearly distinct from the remainder, as were the isolates from cases 3 and 6 . The HIP IS 6110 profiles of the isolates from cases $2,4,5,7$, and 8 were indistinguishable, and these were considered to be representatives of a single outbreak strain. Repeated analysis demonstrated identical profiles.

\section{Discussion}

This study shows the value of molecular typing of $M$ tuberculosis isolates from a putative outbreak. Although the first case subsequently proved to be unrelated to the others, it did trigger contact tracing which revealed a hitherto unsuspected outbreak of tuberculosis among this high risk population. The fact that two further unrelated cases of tuberculosis were also detected is perhaps unsurprising in view of the multiplicity of risk factors in this population group.
These data suggest that hostels for the homeless may be an important focus for transmission of tuberculosis, but contact tracing is difficult in a such a transient population. Nevertheless, people who use such accommodation are likely to be at greater risk of tuberculosis than the general population and this study illustrates some important technical aspects of such investigations.

One of the most useful genetic markers of the $M$ tuberculosis complex is IS6110. The chromosomal copy number and location of this element varies between strains, resulting in a high degree of polymorphism. Consequently, IS6110 profiling has been used for the comparison of isolates of $M$ tuberculosis and has been shown to be highly discriminatory. ${ }^{37}$

The standard IS6110-RFLP typing method, although discriminatory and reproducible, is laborious and requires several micrograms of chromosomal DNA. ${ }^{68}$ In contrast, HIP is technically simple and requires considerably less DNA than the standard RFLP method. Nevertheless, HIP has been shown to have the reproducibility and discriminatory ability of standard IS6110-RFLP typing, ${ }^{8}$ the size and number of PCR amplicons produced depending on both the number of copies and sites of integration of IS6110.

In this study, the IS6110 profiling using the PCR method was rapid (with two rounds of amplification being completed in around one hour using a Rapidcycler), and required fewer manipulations than the standard RFLP method, which is based on a Southern blotting technique. HIP profiling has previously been successfully applied to the investigation of an outbreak of tuberculosis, ${ }^{9}$ and the results presented here support and extend this finding. The HIP method enabled DNA extracted from isolates during the evolution of an outbreak to be archived and then recalled without further processing for immediate testing alongside DNA from new isolates. Alternatively, profiles can be stored on computer disk and compared with new profiles at a later date using a suitable software package.

HIP proved particularly valuable in the outbreak reported here because the isolates were recovered at intervals over a period of 28 months, and initial RFLP analysis was poorly discriminatory. In this outbreak, the chain of potential contacts was followed over a protracted period during which positive cases were presumably resident in other hostels. It is clear that transmission of infection occurred among these patients, but unclear which was the index case, or indeed whether a single index case was involved. Suspicion must fall on case 2, however, as this yielded the first of the outbreak strains and predated other isolates. Interest- 
ingly, this patient was smear negative, ${ }^{10}$ although the possibility of an another undetected smear positive individual being the index case cannot be discounted. Contact tracing is continuing and it is quite likely that other cases of tuberculosis will be identified. Any further isolates of $M$ tuberculosis can readily be compared with those of the outbreak strain, using the stored DNA extracts.

We conclude that HIP typing of $M$ tuberculosis isolates from putative outbreaks may provide significant advantages over RFLP typing, particularly when the outbreak under investigation is of a protracted nature.

We thank the Communicable Disease Control Unit, General Hospital, Newcastle upon Tyne, in particular Ms Elspeth Hospital, Newcastle upon Tyne, in particular Ms Elspeth
Gould, for the provision of contact tracing and epidemiological data.

1 Watson JM. Tuberculosis in Britain today. $B M \mathcal{F}$ 1993;306:221-2.
2 Kumar D, Citron KM, Leese J, et al. Tuberculosis among the homeless at a temporary shelter in London: report of a chest X-ray screening programme. $\mathcal{F}$ Epidemiol Community Health 1995;49:629-33.

3 Saunders NA. State of the art: typing Mycobacterium tuberculosis. F Hosp Infect 1995;29:169-76.

4 Patel S, Wall S, Saunders NA. Heminested inverse PCR for IS6110 fingerprinting of Mycobacterium tuberculosis strains. F Clin Microbiol 1996;34:1686-90.

5 Kent PT, Kubica GP. Public Health Mycobacteriology: a Kent PT, Kubica GP. Public Health Mycobacteriology: a
guide for the level III laboratory. Atlanta, Georgia: US guide for the level III laboratory. Atlanta, Georgia: US Department of Health
Disease Control, 1985.

6 van Embden JDA, Cave MD, Crawford JT, et al. Strain identification of Mycobacterium tuberculosis by DNA fingerprinting: recommendations for a standardized methodology. F Clin Microbiol 1993;31:406-9.

7 Breathnach AS, de Ruiter A, Holdsworth GMC, et al. An outbreak of multi-drug-resistant tuberculosis in a London teaching hospital. F Hosp Infect 1998;39:111-17.

8 Saunders NA. Strain typing of Mycobacterium tuberculosis. F Infect 1999;38:80-6.

9 Saunders NA, Metherell L, Patel S. Investigation of an outbreak of multidrug resistant tuberculosis among renal patients using $\mathrm{rpoB}$ gene sequencing and IS6110 inverse PCR. F Infect 1997;35:129-33.

10 Behr MA, Warren SA, Salamon H, et al. Transmission of Mycobacterium tuberculosis from patients smear-negative for acid-fast bacilli. Lancet 1999; 353:444-9. 